SALUD

Ciencias sociales

HUMANIDADES

LA NEUROÉTICA Y LA POSIBILIDAD DE LA MEJORA HUMANA

PENSAMENT ACTUAL

SILENCIO EN EL RUIDO: OPORTUNIDADES PARA LA MEDITACIÓN EN LA VIDA COTIDIANA -SALVADOR CASADO

ARTE, SALUD Y SOCIEDAD

UN ENCARGO SOBRE PLA-NARBONA

-MARIA LEON SANROMȦ 


\section{Co-directores}

Marc Antoni Broggi i Trias (PCBC)

Francesc Borrell (UB)

Jefa de Redacción

Núria Estrach (UAB)

\section{Consejo científico}

Juan Carlos Hernández Clemente

Juan Medrano Albéniz

Vicente Morales Hidalgo

\section{Correspondencia}

Web:

http://www.fundacionletamendi.com

Correo electrónico:

info@fundacionletamendi.com

Envío de manuscritos:

http://www.fundacionletamendi.com/revista-foliahumanistica/envio-de-manuscritos/

\section{Información editorial}

Folia Humanística publica artículos por encargo solicitados a especialistas, así como aquellas propuestas enviadas por los autores y aceptadas tras su evaluación por pares de académicos especializados.

Los textos recibidos se publicaran en la lengua original (castellano, catalán, inglés y francés); los que se consideren de relevancia mayor serán traducidos al inglés y castellano.

Los artículos deben ser originales y acompañados del documento "derechos de autor" que encontrarán en la web, junto a las normas de presentación a seguir.

Cada artículo publicado al final tendrá especificado la referencia de citación, donde se incluirá el número DOI ${ }^{\circledR}$.

\section{Distribución}

La Revista Folia Humanística es de libre acceso a consultar online.

http://www.fundacionletamendi.com/category/rev istal

Folia Humanística es una revista internacional que tiene el doble objetivo de fomentar, por un lado, la reflexión y el debate público en el ámbito de la Salud, Ciencias Sociales y Humanidades, y por el otro, la colaboración entre distintos equipos de investigación nacionales e internacionales que dinamicen el diálogo entre la filosofía de la medicina, la salud pública y la justicia social. Dividida en "Tema del día", (artículos para el debate), "Pensamiento actual", (artículos críticos de novedades editoriales), y "Arte, Salud y Sociedad", la revista se esfuerza en fortalecer las conexiones entre la investigación académica, la práctica clínica, las experiencias de los pacientes y sus implicaciones éticas y estéticas en la sociedad. Todo ello con la intención de favorecer la reflexión entre diferentes disciplinas sobre temas de actualidad y las tendencias más novedosas en el campo de las Humanidades y la Salud.

Folia Humanística is an International Journal, born with the dual aim of fuelling the discussion and public debate on issues of health, social sciences and humanities and on the hand, of fostering cooperation between various research groups, both national and International, to spur the dialogue between philosophy and medicine, public health and social justice. The Journal is divided into three different sections: "main focus" (article for debate), "Contemporary thought" (critical reviews of new Publications) and "Arts, Health and Society" which all contribute to strengthening the links between academic research, clinical practice, the experience of patients and their ethical and esthetical implications for society. Ultimately, the intention of the Journal is to promote reflection at the crossroads of several disciplines on topical issues and new trends in humanities and health. 


\title{
EL SILENCIO EN EL RUIDO: OPORTUNIDADES PARA LA MEDITACIÓN EN LA VIDA COTIDIANA
}

\section{Salvador Casado}

\begin{abstract}
Resumen: Los factores que condicionan el aumento del ruido de nuestra época son: el urbanismo, la tecnología y los medios de comunicación. La sociedad del conocimiento nos provee de una sobrecarga informativa en forma de ruido de fondo que nos produce desasosiego. Para poder observar y manejar esta desazón se precisa inteligencia intrapersonal y capacidad para generar silencio de calidad. El mundo sanitario y académico son ejemplos de profesiones donde el ruido puede ser devastador. Para poder manejarlo es importante aprender a desarrollar sensibilidad y atención hacia el exterior por un lado y hacia uno mismo por otro. En las crisis vitales es donde solemos aprender a acercarnos al espacio interior de silencio. El sufrimiento personal nos obliga a buscar soluciones y para ello no hay otro remedio que terminar mirando hacia donde señalan las emociones, por muy incómodo o difícil que nos resulte.
\end{abstract}

El proceso de digitalización de la comunicación ha aumentado el "ancho de banda" de información que recibimos y nos obliga a gastar mucha energía para manejarlo. Recibimos más estímulos de los necesarios, mientras que en paralelo la sociedad sufre una epidemia de soledad y aislamiento sin precedentes que afecta a niños, adultos y ancianos. Concluimos que la sobrecarga informativa produce sufrimiento. Dado que la sociedad actual produce mucho ruido es importante aprender a generar silencio y a relacionarnos con él, cuidando los espacios de silencio personal e implementando actitudes meditativas que mejoren el contacto interior y las relaciones humanas de calidad. La calibración y afinamiento personal y profesional precisan pues de espacio vacío por lo que aprender a equilibrar el ruido y el silencio en nuestra vida es un reto vital para todos.

Palabras clave: meditación/ agotamiento profesional/ atención plena/ estrés psicológico/.

Abstract: THE SILENCE IN THE NOISE: OPPORTUNITIES FOR MEDITATION IN EVERYDAY LIFE.

The increase of noise in our era is conditioned by three factors: urbanism, technology and the media. The knowledge society provides us with an information overload in the form of background noise that causes us discomfort. To be able to observe and manage this discomfort requires intrapersonal intelligence and the ability to generate quality silence. The sanitary and teaching worlds are examples of professions where noise can be devastating. In order to be able to manage it, it is important to learn to develop sensitivity and attention to the outside on one side and to oneself on the other. It is in vital crises that we usually learn to approach the interior space of silence. Personal suffering forces us to seek solutions and for this there is no alternative but to end up looking towards where emotions point, no matter how uncomfortable or difficult it may be.

The process of digitizing communication has increased the "bandwidth" of information we receive and forces us to spend more energy to manage it. We are receiving more stimuli than necessary while at the same time society is experiencing an unprecedented epidemic of loneliness and isolation affecting children, adults and the elderly.We conclude that information overload causes suffering. Given that today's society produces a lot of noise, it is important to learn how to generate silence and relate to it, taking care of the spaces of 
personal silence and implementing meditative attitudes that improve inner contact and quality human relations. Personal and professional calibration and tuning therefore require empty space, so learning to balance noise and silence in our lives is a vital challenge for everyone.

Keywords: meditation/ professional exhaustion/mindfulness/ psychological stress/.

Artículo recibido: 18 febrero 2018; aceptado: 30 julio 2018.

\section{INTRODUCCIÓN}

Somos hijos de un tiempo y el nuestro es un tiempo de ruido ${ }^{1}$. Aunque el proceso civilizador acelera la capacidad humana para manipular y dominar el medio una de las consecuencias es el deterioro y la contaminación del mismo. Dentro de esa inevitable polución se encuentra el ruido, una constante, insidiosa y persistente que consigue conquistar cada vez más ámbitos. Ibn Jaldún ${ }^{2}$ se quejaba en el siglo $\mathrm{XVI}$ de la insufrible suciedad y jaleo de las ciudades de su tiempo. Queja que podemos escuchar en eruditos de toda época y cultura; hoy devenida un clamor. Lo cierto es que aborrecemos este ruido, pero no somos capaces de zafarnos de él; pues vivimos en una sociedad de la información que nos escupe datos a granel desde que nos levantamos hasta el mismo momento de acostarnos.

Son tres los factores que condicionan este aumento desaforado del ruido: el urbanismo, la tecnología y los medios de comunicación ${ }^{3}$. El urbanismo de nuestras sociedades es un hecho. Las zonas rurales desde hace ya tiempo que se despueblan $^{4}$, en busca de mayores oportunidades, y las ciudades, cada vez mayores, acumulan incontables vehículos a motor para garantizar la movilidad. Los ritmos de vida rápidos, la inversión de mayor tiempo para los desplazamientos, la acumulación de personas y coches hacen que la urbe sea un lugar ruidoso y contaminado.

\footnotetext{
${ }^{1}$ Kagge, E. (2017) El silencio en la era del ruido: El placer de evadirse del mundo. Penguin Random House.

${ }^{2}$ Braudel, F. (2015) El Mediterráneo y el mundo mediterráneo en la época de Felipe II. Tomo 2. Fondo de cultura económica.

${ }^{3}$ Mcluhan, E. La teoría de la comunicación de Marshall McLuhan: el butronero. Palabra Clave, 2015; 18 (4).

${ }^{4}$ Del Molino, S. (2016) La España vacía. Turner.
} 
En cuanto a la tecnología, la ley de Moore $^{5}$ nos dice que cada dos años se duplica la capacidad de computación de los ordenadores. Es un ejemplo claro de la velocidad de mejora de la tecnología que pone en nuestras manos equipos y aparatos con capacidades cada vez más potentes y sofisticadas. Los teléfonos móviles integran funciones que, previamente, detentaban los ordenadores domésticos, además de ofrecer nuevos servicios y posibilidades. Nos vamos rodeando de aparatos, servicios y programas que nos ofrecen más operatividad y capacidades, pero que nos cobran un alto precio por el ruido de fondo y las distracciones que producen. Es paradójico que mientras más aplicaciones tenemos en el móvil, menor sea el número de las que realmente usamos, otro ejemplo de ruido de fondo.

Los medios de comunicación de masas tradicionales ${ }^{6}$ se vieron complementados con los digitales derivados de Internet. Hoy cada ciudadano se convierte en productor de sus propios contenidos, y receptor del de su correspondiente red de contactos. Las redes sociales y aplicaciones de mensajería multiplican la comunicación personal, superando en mucho el papel que prestaban antes el correo ordinario, el electrónico y el teléfono. La constante recepción de notificaciones y vigilancia del móvil ${ }^{7}$, en paralelo al resto de pantallas que usamos cotidianamente, es un hecho. La sociedad red multiplica la creación y el movimiento de la información, sobrecargando a la ciudadanía que la produce y consume.

Desde hace tiempo, la sólida sociedad agrícola e industrial se funde con la revolución digital ${ }^{8}$ que convierte en líquidos ${ }^{9}$ los procesos que antes eran rígidos, estancos y estables. Zygmun Bauman ${ }^{10}$ fue el primero en reflexionar sobre este

\footnotetext{
${ }^{5}$ Present, I. Cramming more components onto integrated circuits. Readings in computer architecture, 2000; vol. 56.

${ }^{6}$ Islas, O. Marshall Mcluhan y la complejidad digital. Razón y palabra, 2008; 13 (63).

7 Boase, J.; Ling, R. Measuring mobile phone use: Self report versus log data. Journal of Computer Mediated Communication, 2013; 18 (4): 508-519.

${ }^{8}$ https://es.wikipedia.org/wiki/Revoluci\%C3\%B3n Digital

${ }^{9}$ Vásquez Rocca, A.. (2008) Zygmunt Bauman: modernidad líquida y fragilidad humana. Universidad Complutense de Madrid.

${ }^{10}$ Bauman, Z.(2015) Modernidad líquida. Fondo de cultura económica.
} 
hecho que condiciona una de las principales razones que justifican la coyuntura de crisis que vivimos: todo está cambiando a gran velocidad y nos obliga a adaptarnos al límite de nuestras posibilidades.

Por otro lado la exaltación de la tecnología, el Big Data y la Inteligencia Artificial prometen una progresiva automatización de procesos, profesiones y servicios. Todo lo que sea susceptible de ser automatizado lo será. De esta forma se prescinde de puestos de trabajo y de la implicación humana en los procesos de construcción social creando otra paradoja: ¿Qué papel jugarán los humanos en una sociedad postdigital?

Afrontamos un reto mayúsculo para dar cauce a la primigenia necesidad de silencio que tiene todo ser vivo, fundamental para gozar de un suficiente grado de serenidad y equilibrio. Pareciera que para conseguirlo hemos de nadar contracorriente. Sirva esta reflexión para detenernos un instante, tomar conciencia de nuestra relación con el ruido y el silencio, y la posterior calibración de la misma si hubiera lugar.

\section{LA NECESIDAD DE SILENCIO}

Por mucho que el ser humano sofistique su medio no podrá zafarse de la necesidad de descansar y dormir. Para ello se requiere retomar el silencio que la naturaleza imprime a sus ritmos. Hay un tiempo para la acción y la vigilia, en el que reinan la luz y el sonido, y otro para el descanso y el sueño, en el que prevalecen la oscuridad y el silencio. Este último se reduce artificialmente con el desarrollo de sistemas de iluminación e información que dilatan las horas productivas. No en vano padecemos una epidemia de trastornos del sueño en una época, sobrepasada de estímulos, en la que muchos presumen equivocadamente que es posible desconectar la actividad mental, como si se tratara de otro aparato doméstico más susceptible de apagarse pulsando un botón.

El alejamiento de los ritmos naturales, condicionado por el modo de vida urbano contemporáneo, nos sumerge en un baño de estímulos visuales y auditivos 
durante todas las horas de vigilia, lo que termina produciendo fatiga y cansancio. Hay monitores de televisión en las salas de espera, pasillos, vehículos de transporte público... caso de que el ciudadano no tenga el suyo personal conectado. La sobreabundancia de información modifica nuestro consumo de la misma, prevaleciendo su adicción e ingesta desproporcionada. La obesidad informativa es un hecho poco visibilizado fruto del abuso de fuentes y canales de información que llegan a nosotros a través de pantallas y aparatos, a los que cada vez estamos más expuestos.

En el milenario texto indio Majabharata ${ }^{11}$ se nos muestra el diálogo del arquero Arjuna con su auriga antes de comenzar una batalla. Los dos ejércitos aguardan en formación, dilatando un silencio que se romperá con el combate. Arjuna no desea comenzar el ataque; dado que, en las filas enemigas, hay familiares a los que no quiere dañar. Sabe que del inevitable ruido, inherente a la lucha, surgirá una gran cantidad de dolor y sufrimiento. En las tradiciones religiosas de todas las culturas tenemos ejemplos como este que tratan la dificultad humana para enfrentarse al ruido de la vida. Desde la revolución neolítica el nacimiento de la agricultura modificó la relación del ser humano con el medio, con sus congéneres y con sus dioses. El ruido de fondo ha aumentado desde entonces, propiciando el desarrollo de habilidades para enfrentarse al mismo.

Al igual que se hizo con todo territorio inexplorado, con respecto al silencio también se enviaron exploradores. Los chamanes de los pueblos cazadores recolectores primero, los sacerdotes y ermitaños de las civilizaciones agrarias después, todos abrieron el camino de la búsqueda del silencio ayudados de sustancias químicas, música y técnicas místicas y ascéticas. Las diversas formas de monacato de las distintas tradiciones propusieron reglas de vida que aunaban la actividad y la contemplación. En la cultura europea destaca el ora et labora de la

\footnotetext{
${ }^{11}$ Sukthankar, V. S. (1927) The Mahābhārata: for the first time critically edited.
} 
regla de San Benito, ${ }^{12}$ pero también disponemos de ejemplos en la India del siglo $\mathrm{V}$ antes de la era común.

Uno de los caminos para avanzar en la consecución del silencio interior es la meditación. Sus inicios surgieron de la esfera religiosa, pero posteriormente se abrieron posibilidades laicas no asociadas a sistemas creenciales dentro de la filosofía, las ciencias y las artes. Marià Corbí ${ }^{13}$ defiende con sentido el valor de rescatar estas tradiciones para incluirlas en nuestra sociedad laica, aunque sin la capa de dogmas o ideología que las contuvieron en su tiempo. Como ejemplo citaríamos el uso del yoga o el Mindfulness que son opciones muy sencillas y populares de abrirse a una opción meditativa aconfesional.

Por meditación ${ }^{14}$ consideramos en este escrito todo proceso que ayude a la reflexión, contemplación, pensamiento, oración... y nos permita relacionarnos con el vacío y el silencio, así como con lo que se esconde detrás de estos: la intuición, la revelación, el sentido y la creatividad.

La meditación puede ser formal, cuando sigue alguna técnica de aquietamiento que implique dedicar un tiempo al desarrollo de la misma, o informal, cuando se realiza aportando atención plena, concentración o contemplación ante una actividad cotidiana como pasear ${ }^{15}{ }^{16}$, observar detenidamente un objeto 0 realizar una acción de forma cuidadosa.

Existen múltiples posibilidades de meditación formal que provienen de diversas tradiciones espirituales y que pueden ser usadas dentro o fuera de esa tradición. Así sucede en Occidente con la práctica común de la meditación tipo yoga, respiración consciente o contemplación de un objeto fuera de toda creencia o culto religioso determinado. La psicología moderna también nos provee de un buen

\footnotetext{
${ }^{12}$ http://www.britannica.com/EBchecked/topic/388935/monasticism

13 Quiñonero, M.C. (2010) Hacia una espiritualidad laica: sin creencias, sin religiones, sin dioses. Herder Editorial.

${ }^{14}$ Prieto, J.M. Psicología de la meditación, la psique de vuelta a casa. InfocopOnline-Revista de Psicología, 2007; (33).

${ }^{15}$ Le Breton, D. (2015); Elogio del caminar. Siruela.

${ }^{16}$ Schelle, K.G.; Silvestre López, F.; Hernández, I. (2013) El arte de pasear. Díaz \& Pons.
} 
conjunto de técnicas de meditación ${ }^{17}$, centradas en la relajación corporal, la tranquilización del ánimo o el control del estrés. La visualización o imaginación creativa es otro campo que permite focalizar la atención con diversos objetivos.

La meditación informal puede rescatarse en el seno de cualquier actividad. Un tiempo de silencio en el que concentramos la mente en un pensamiento, problema o asunto es lo más habitual. También es posible centrar la atención en la acción que ocupe el presente de la persona, de este modo actividades cotidianas como el aseo, la ingesta o la limpieza se pueden dotar de plena conciencia lo que silencia el ruido interior del pensamiento errante. Los estados de flujo ${ }^{18}$ que se producen al realizar una acción de forma virtuosa también consiguen silenciamiento interior.

La evidencia científica nos dice que dedicamos un 49\% del tiempo de vigilia a pensamiento $\sin$ foco $^{19}$. Esto se logra a base de automatizar procesos. De este modo mientras conducimos o hacemos actividades rutinarias nuestra mente vaga a su placer por pensamientos, recuerdos o fabulaciones de futuro. Este tipo de pensamiento es espoleado por la sobrecarga cognitiva de estímulos externos, por lo que se puede decir que el ruido externo genera ruido interno.

\section{LA MEDITACIÓN COMO CAMINO PERSONAL}

Nuestra cultura no favorece la capacidad natural para acercarnos al silencio. Los que se han aventurado alguna vez mar adentro ${ }^{20}$ o han atravesado algún desierto lo conocen, pero suele ser común no separarse mucho de la costa ni adentrarse mucho en descampado. Lo mismo se aplica a las extensas superficies interiores. Nos mantenemos cerca de las fuentes de ruido que de alguna forma nos dan seguridad, aunque nos cansen y sobrecarguen. Pagamos sin duda un precio por

\footnotetext{
${ }^{17}$ Campagne, D.M. Teoría y fisiología de la meditación. Cuadernos de medicina psicosomática y psiquiatría de enlace, 2004; 69 (70): 15-30.

${ }^{18}$ Csikszentmihalyi, M. (2012). Fluir: una psicología de la felicidad. Editorial Kairós.

${ }^{19} \mathrm{http}: / /$ www.elmundo.es/elmundosalud/2010/11/11/neurociencia/1289481239.html

${ }^{20}$ LC 5, 1-14
} 
ello, pero no nos importa. El miedo y el horror que produce el vacío bien merece ese coste, aunque sea alto.

Es común que todos nos vayamos llenando de problemas, situaciones o asuntos a los que damos vueltas de forma constante ${ }^{21}$, sin ser capaces de tomar una decisión para solucionarlos. Este tipo de situaciones producen ruido interno que se suma al quantum de ruido externo que soportamos y que en ocasiones lo supera con creces. Analizaremos más adelante con detalle lo que sucede en las crisis vitales y ofreceremos cursos de acción alternativos.

Dado que el ruido interno suele ser más fastidioso que el externo, es frecuente que tratemos de distraerlo aumentando el segundo. Solemos relacionarnos con nuestras dificultades huyendo o reprimiéndolas, usando para ello todo tipo de distracciones.

Para poder observar y manejar la desazón interior se precisa de inteligencia intrapersonal, siguiendo la teoría de inteligencias múltiples de Gardner ${ }^{22}$. Con observación introspectiva es posible detectar el pensamiento o la emoción que nos incomoda y, aplicando consciencia, ser capaces de relacionarnos con ella sin reaccionar.

Las técnicas de atención plena o Mindfulness llevan años proporcionando ayuda para conseguir crear espacio y silencio interno, punto de arranque imprescincible para podernos manejar en el territorio intrapersonal. El profesor Jon Kabat Zinn ${ }^{23}$ aplicó un programa para la reducción de estrés de pacientes ingresados usando este enfoque. Sus buenos resultados ${ }^{24}$ han hecho que se

${ }^{21}$ Delespaux, E., Zech, E. Déficits de inhibición cognitiva y rumiación posterior a una pérdida: evidencia a partir de una tarea de supresión de pensamientos. Estudios de Psicología, 2017; 38 (3): 622-638.

${ }^{22}$ Gardner, H.E. (2008); Multiple intelligences: New horizons in theory and practice. Basic books.

${ }^{23}$ Kabat-Zinn, J. (2004) Vivir con plenitud las crisis. Cómo utilizar la sabiduría del cuerpo y de la mente para afrontar el estrés, el dolor y la enfermedad.

${ }^{24}$ Grossman, P. et al. Mindfulness-based stress reduction and health benefits: A meta-analysis. Journal of psychosomatic research, 2004; 57 (1):35-43. 
popularicen sobremanera, lo que pone el foco en la profunda necesidad humana para encontrar sosiego en un mundo agitado y ruidoso.

Mucho antes que Kabat Zinn, los exploradores que las distintas tradiciones enviaron al desierto del silencio, regresaron con valiosos mapas y conocimientos. Había vida y serenidad en la sequedad y dureza del desierto, encontraron tesoros que nos seguirán resultando muy valiosos.

Cada cual suele ensayar aquellas técnicas meditativas que ve en su ámbito familiar o cultural. A esto se suman las numerosas opciones y corrientes que la globalización nos pone encima de la mesa, lo que ha creado un nuevo mercado de productos de espiritualidad y trascendencia que es una prolongación del de la autoayuda.

En el momento actual se está estudiando si la aplicación de técnicas de meditación en niños ${ }^{25}$ pudiera ser de utilidad. No cabe duda de que facilitar que los jóvenes manejen mejor el exceso de ruido y aprendan a generar silencio parece una buena inversión ${ }^{26}$.

Desde siempre han existido rutinas generadoras de silencio, como dar un paseo. Son legión los autores que han encontrado tranquilidad, sosiego e inspiración por este método.

La retirada parcial del mundo es otra opción. Algunos utilizan su tiempo de vacaciones para ello, otros como Thoreau ${ }^{27}$ decidieron pasar largas temporadas buscando la simplicidad y el silencio. Pese a que las dinámicas vacacionales suelen cambiar un tipo de ruido por otro, cada vez existen más opciones de turismo tranquilo, lento o silencioso. Por otro lado cada vez hay más personas que deciden

${ }^{25}$ Linden, W. Practicing of meditation by school children and their levels of field dependenceindependence, test anxiety, and reading achievement. Journal of Consulting and Clinical Psychology, 1973; 41 (1): 139.

${ }^{26}$ Burke, C.A. Mindfulness-based approaches with children and adolescents: A preliminary review of current research in an emergent field. Journal of child and family studies, 2010; 19 (2):133-144.

${ }^{27}$ Thoreau, H.D. Walden, or Life in the Woods. 1854. Minneola, NY: Dover, 1995. 
tomarse un tiempo sabático para encontrase con ellos mismos o reordenarse interiormente.

La tradición de los padres del desierto ${ }^{28}$ en Egipto es paralela a la de muchas culturas, en las que el sabio se retiraba a la naturaleza para encontrar una iluminación que el ruido de la proximidad humana dificulta. La ascética y la mística ${ }^{29}$ se englobarían en este capítulo. Dentro del mundo del arte muchos creadores han buscado fuentes de creatividad en el silencio y la naturaleza. Los pintores paisajistas gustan de pintar al natural, para estar en pleno contacto con lo que quieren plasmar. La leyenda de la manzana de Newton también aplica este principio al área científica. Thomas Edison popularizó la hipnagogia ${ }^{30}$ que buscaba la inspiración en el límite entre la vigilia y el sueño. De este modo se quedaba traspuesto en un sillón mientras sostenía una bola metálica en la mano. Al quedarse dormido la bola caía despertándolo con un ruido. En ese momento trataba de pescar lo que su conciencia aun recordara. Otros han investigado los sueños ${ }^{31}$ como fuente de inspiración, tratando de recordar los mismos para después analizarlos. Existen pues incontables caminos meditativos $^{32}$ que buscan acercarse y contemplar diversas realidades. Son formas de hacer silencio en el ruido cotidiano que están a disposición de quien las necesite.

\section{LA MEDITACIÓN EN LAS PROFESIONES SANITARIAS}

El ejercicio de la medicina ha sufrido modificaciones a lo largo de la historia. En el momento actual afronta un cambio radical al enfrentarse a una crisis global sin precedentes que la afecta de lleno.

\footnotetext{
${ }^{28}$ Grün, A.; García, P. (2003) La sabiduría de los padres del desierto. Salamanca.

${ }^{29}$ Rossi, R. (2010) Juan de la Cruz: silencio y creatividad. Editorial Trotta.

${ }^{30}$ De Bustamante Vega, M., et al. (2016); Introducción al sueño creador de arquitecturas. El sueño de la razón VS. el sueño de la intuición.

${ }^{31}$ Freud, S. (2013); The interpretation of dreams. Read Books Ltd.

${ }^{32}$ Eliade, M. (1972); Tratado de historia de las religiones. Ediciones Era.
} 
El aumento de carga de enfermedad por el envejecimiento poblacional y en complejidad, al entremezclarse problemas de salud con problemas sociales y de otra índole, condiciona que la capacidad de respuesta de los sistemas sanitarios disminuya. Por otro lado, dentro de las personas sanas, cada vez es menor la tolerancia a la dificultad y al malestar siendo progresivamente más frecuente y normal consultar a algún profesional sanitario ante la más mínima molestia.

Este contexto está produciendo una sobrecarga creciente en todas las profesiones sanitarias, tanto de gestión privada como pública. Al final se obliga al profesional a ver a más pacientes en menos tiempo, disminuyéndose las posibilidades de crear encuentros clínicos significativos ${ }^{33}$ en los que fluya una comunicación de calidad.

Al tiempo que atiende al paciente, hoy el profesional médico debe atender al teléfono, a la pantalla de ordenador $\mathrm{y} / \mathrm{o}$ a otros colegas. Ese aumento de interrupciones ${ }^{34}$ junto al ruido de fondo que produce los sistemas de información y de historial clínica electrónica (diseñados más a criterio de los gestores o ingenieros informáticos que del uso clínico) va en detrimento sin duda del paciente que tiene delante: pues disminuye la necesaria concentración médica y propicia los errores.

En un desempeño clínico clásico, tras la anamnesis y exploración del paciente se debe disponer de un tiempo de reflexión ${ }^{35}$ de calidad del caso; un tiempo que permita analizarlo, entender su mecanismo específico, para luego elaborar un diagnóstico certero $\mathrm{y}$, en consecuencia, un tratamiento adecuado. Cuando la complejidad del caso es alta con mayor motivo. No disponer de este espacio de reflexión sería parejo a disminuir los silencios de una partitura. Al final el intérprete tocaría música acelerada sin ninguna armonía.

\footnotetext{
${ }^{33}$ Girón, M. et al. Calidad de la relación médico paciente y resultados de los encuentros clínicos en atención primaria de Alicante: un estudio con grupos focales. Revista Española de Salud Pública, 2002; 76: 561-575.

${ }^{34}$ Vázquez, M.T. Jubete, et al. Estudio de la calidad de vida profesional en los trabajadores de atención primaria del área 1 de Madrid. Atención Primaria, 2005; 36 (2): 112-114.

35 Borrell-Carrió, F.; Hernández-Clement, J.C. Reflexión en la práctica clínica. Revista Clínica Española, 2014; 214 (2): 94-100.
} 
Tras pasar de forma habitual una consulta de más pacientes de los que serían aconsejables, es normal que los profesionales de la salud pierdan calibración y puedan empezar a sufrir algún signo de desgaste profesional ${ }^{36}$.

Es importante que cada profesional de la salud se analice y se evalúe para detectar si aparecen señales de malestar o de desgaste. Dado que no es habitual tener un consultor, psicólogo o grupo de ayuda (tipo Balint) será necesario un cambio de cultura personal que, de momento, no está facilitando la estructura. Un profesional que salga agotado y derrotado de su consulta de forma habitual, debería ser capaz de tomar conciencia para evitar daños personales y ajenos.

Para crear silencio en el ruido sanitario es fundamental la correcta calibración. Y para ésta es importante aprender a desarrollar una sensibilidad y atención hacia el paciente, por un lado, y hacia el propio profesional, por otro; como Roger Neighbour ${ }^{37}$ nos enseñó en su excelente libro La consulta interior.

Poner el foco en crear encuentros clínicos de calidad basado en una comunicación clínica esmerada de los que salgan satisfechos tanto pacientes como profesionales es básico. Poco se insiste en el papel de la comunicación en el mundo sanitario, pero hay que recordar que es un elemento de primer nivel en toda asistencia que quiera ser de calidad ${ }^{38}$.

Ya en el siglo pasado muchos médicos se dieron cuenta del componente de desgaste personal que produce el contacto permanente con la enfermedad y el sufrimiento humano. Trataron de defenderse desde posturas humanistas, en las que compatibilizaban su ejercicio sanitario con actividades como la escritura o las bellas $\operatorname{artes}^{39}$. Tocar un instrumento musical, pintar o dedicarse a la literatura eran tareas comunes para muchos. Hoy no es práctica tan extendida al haber cambiado mucho

\footnotetext{
${ }^{36}$ Mingote Adán, J.C.; Moreno Jiménez, B.; Gálvez Herrer, Mónica, Desgaste profesional y salud de los profesionales médicos: revisión y propuestas de prevención. Medicina clínica, $2004 ; 123$ (7): 265-270.

${ }^{37}$ Neighbour, R. (1987); The inner consultation. Lancaster: MTP press.

38 Leonard, M; Graham, S. Bonacum, D.. The human factor: the critical importance of effective teamwork and communication in providing safe care. BMJ Quality \& Safety, 2004;13 (suppl 1): 185190.

${ }^{39}$ Hurwitz, B. Medicine, the arts and humanities. Clinical Medicine, 2003; 3 (6):497-498.
} 
los tiempos, aunque debería rescatarse la reflexión sobre la idoneidad de animar a todo profesional de la salud a desarrollar estas facetas creativas ${ }^{40}$.

Estas actividades de calibración externa se basan en rescatar el cuidado de espacios de silencio de los que pueda surgir creatividad, inspiración y belleza. Ingredientes capaces de diluir el potente ácido que la dificultad humana ajena lanza sobre los que profesionalmente se dedican a cuidar. Esto por supuesto es exportable a todo aquel que cuide a algún familiar enfermo o discapacitado. Todo cuidador ha de estar equilibrado si quiere cuidar bien.

\section{LA MEDITACIÓN EN LA ESFERA EDUCATIVA}

Los profesionales de la educación se han relacionado desde siempre con las nuevas generaciones. Uno de los signos de identidad de las actuales es su nivel de ruido ${ }^{41}$ y agitación, sometidos como están desde que nacen a un ambiente hiperestimulante donde no paran de estar sometidos a pantallas y ruido.

Conseguir la atención de un grupo de jóvenes es hoy un reto mayúsculo, acostumbrados como están a pasar varias horas al día consumiendo material audiovisual mucho más atractivo y estimulante que una clase magistral con apoyo en pizarra o en presentación de diapositivas. El aparente desinterés de la mayoría dificulta que el profesor llegue a transmitir con calidad, dificultando así también la relación con los pocos alumnos interesados; hecho que genera espirales de tensión que resienten el ambiente y el rendimiento académico general.

Por todo ello los profesores sufren un alto nivel de desgaste profesional ${ }^{42}$ que condiciona desgaste profesional, bajas laborales y gran carga de sufrimiento.

\footnotetext{
${ }^{40}$ Shapiro, J.; Rucker, LI. Can poetry make better doctors? Teaching the humanities and arts to medical students and residents at the University of California, Irvine, College of Medicine. Academic Medicine, 2003; 78 (10): 953-957.

${ }^{41}$ Collado Martínez, J. M.. La visión del profesorado de Secundaria sobre la contaminación acústica. Nuevas tendencias en investigaciones en Educación Ambiental, 2004; 27.

${ }^{42}$ Moriana Elvira, J. A.; Herruzo Cabrera, J. Estrés y burnout en profesores. International journal of clinical and health psychology, 2004; 4 (3)
} 
El valor del silencio no se ejerce ni se enseña por los sistemas educativos modernos. Tampoco los profesores saben como protegerse de la enorme carga de ruido que su profesión implica. Al igual que pasaba en la esfera sanitaria es común que esta situación de sobrecarga y desgaste termine haciendo mella en quien la padece, desafinándole personal y profesionalmente. Al final las conductas evitativas, de eludir responsabilidades o de mínimos hacen que la calidad de su enseñanza se resienta en un marco en el que todos terminan perdiendo.

Dentro de la comunicación que establece un docente con sus alumnos es crucial la parte no verbal ${ }^{43}$. Si el profesor no está bien afinado, sus discentes lo notarán enseguida. El verdadero profesor lo sabe, por ello cuida su espacio interior para manejar las preocupaciones y ruidos que pueda haber y no trasladarlos al exterior en su ejercicio profesional.

Como veíamos que pasaba en el ámbito sanitario, en el mundo académico es necesaria una acción institucional que proteja a los profesionales; una acción que vaya en paralelo a la que estos mismos puedan implementar en si mismos. Incentivar la formación a este nivel y estimular la creación de redes profesionales, grupos de apoyo o comunidades locales es otro curso de acción de gran valor.

\section{CRISIS VITALES Y SILENCIO}

Toda crisis vital se caracteriza por hacernos perder pie. Afrontar una pérdida o circunstancia estresante de calado nos vuelve vulnerables como la ola que nos revuelca y nos empuja debajo de las aguas. Perdemos la orientación y la estabilidad, somos zarandeados por potentes fuerzas que no podemos controlar y, lo peor de todo, sentimos que no podemos respirar, que nos ahogamos ${ }^{44}$.

El sufrimiento personal nos obliga a buscar soluciones y para ello no hay otro remedio que terminar mirando hacia donde señalan las emociones, por muy

\footnotetext{
${ }^{43}$ Ajuria, H. A. De A.. Influencias de la comunicación no verbal en los estilos de enseñanza y en los estilos de aprendizaje. Revista de educación, 2004; (334): 21-32.

${ }^{44}$ Erikson, E. H. (2012); El ciclo vital completado: Edición revisada y ampliada. Grupo Planeta Spain.
} 
incómodo o difícil que nos resulte. Cuando la tristeza, el miedo, la ansiedad o el asco son muy intensos solemos tratar de escapar y distraernos o de luchar y empujar la emoción fuera de la vista. Pero si la esfera emocional es muy intensa no nos resultará posible, por mucho ruido que tratemos de hacer para no tener que atender lo evidente.

La crisis vital ${ }^{45}$ lleva implícita la convivencia con el ensordecedor ruido de las alarmas interiores emocionales y cognitivas que nos avisarán vehementemente de la emergencia vital que encaramos. La intensidad de decibelios interiores suele superar todos nuestros vanos intentos de huida y termina postrándonos en un estado de impotencia que se suele describir como estar en el pozo o en un callejón sin salida.

Al final la cura o solución nos espera en el espacio vacío y silencioso de nuestro desierto interior, precisamente en el lugar que en lo cotidiano tratamos de evitar vehementemente. Será necesario atravesar un tiempo de duelo o adaptación cuya duración dependerá de factores intrínsecos y extrínsecos.

Uno solo consigue superar la pérdida de un ser querido, la integración de una enfermedad o discapacidad, una ruptura sentimental o cualquier desastre personal solo cuando se atreve a contemplarlo sin escapar ni atacar las emociones, pensamientos o recuerdos que esa situación provoque en nosotros.

Nadie nos enseña a hacerlo. Vamos aprendiendo por ensayo y error a base de ser zarandeados por olas cada vez mayores. Terminamos comprendiendo que ante lo inevitable de la marejada de poco sirve el ruido y la agitación y que, si uno se rinde a la evidencia y se deja hacer, tarde o temprano terminará subiendo a superficie donde podrá respirar de nuevo a voluntad.

\footnotetext{
${ }^{45}$ Fierro, A. (1997), Estrés, afrontamiento y adaptación. Estrés y Salud. Valencia: Promolibro.
} 


\section{ANTE LA SOBRECARGA INFORMATIVA}

La información es hoy parte ineludible de nuestras jornadas. Ya al despertar nos ayudamos de aparatos de radio, televisión o comunicación personal para consultar el oráculo informativo que nos avisa de las últimas novedades o temas de interés. Y terminamos el día mirando nuestro teléfono móvil por si hubiera llegado algún último correo electrónico o mensaje de importancia.

En el ínterin son varias las horas que dedicaremos a relacionarnos con pantallas de todo tipo, además de soportar incontables interrupciones por sonidos, pitidos y avisos que nos reclaman atención ante las notificaciones de las aplicaciones y programas que nos conectan socialmente con los demás.

La tecnología pone hoy en nuestra mano la capacidad de producir información y de recibirla con medios que hace pocos años solo las agencias de comunicación disponían ${ }^{46}$. Esto permite que cualquier ciudadano pueda mantener una red compleja de contactos con la que relacionarse a nivel personal o profesional. Potencialmente cualquier persona puede tener presencia digital y producir contenidos que lleguen virtualmente a cualquier punto del planeta. Así mismo cualquiera puede conectarse con ciudadanos de otras culturas y países para seguir los textos, vídeos o demás material que estos produzcan.

Este proceso de digitalización de la comunicación ${ }^{47}$ ha aumentado el "ancho de banda" de información que recibimos. Del mismo modo que los aparatos electrónicos cada vez aumentan más su capacidad y velocidad de transmisión, sus usuarios de forma paralela se ven obligados a manejar mayor número de fuentes, mensajes y unidades de información en un tiempo dado ${ }^{48}$.

Llegados a un determinado punto es fundamental desplegar filtros ${ }^{49} \circ$ conductas de retirada y apagado de dichos sistemas de comunicación. La conexión

\footnotetext{
${ }^{46}$ Nafría, I. Web 2.0: El usuario, el nuevo rey de Internet. Gestión, 2000, 2007.

${ }^{47}$ Lévy, P.; Medina, M. Cibercultura: la cultura de la sociedad digital. Anthropos, 2007.

${ }^{48}$ Casas-Mas, B. Infoxicación a través de los medios de comunicación. Ámbitos, 2014; (24).

${ }^{49}$ Mena Muñoz, S. Herramientas contra la infoxicación en los Social Media: los "Content Curators". Ambitos, 2014; (24).
} 
continua inunda la conciencia con un insoportable ruido de fondo que puede llegar a dificultar el desempeño de nuestras obligaciones laborales y personales.

Por otro lado para poder desarrollar creatividad requerimos un mínimo de espacio y silencio que implica una separación de los ruidosos canales informativos que nos rodean ${ }^{50}$. Aprender a encender y apagar aparatos, aplicaciones y servicios. Saber qué tiempo dedicar a conectar y a desconectar, a usar unos canales o dejar de usar otros será fundamental tanto para nosotros como para las personas con las que convivamos o nos relacionemos.

\section{SOLEDAD Y RELACIONES SOCIALES}

El ritmo de vida contemporáneo basado en la rapidez y la eficacia nos obliga a dedicar muchas horas al desempeño laboral y otras muchas al transporte y la movilidad. Nos pasamos la vida de casa al trabajo y perdemos espacios públicos de encuentro y socialización. Cada vez se compra y se interactúa más a distancia y se pisan menos las plazas y mercados tradicionales.

Los modelos de urbanismo nos posicionan en urbanizaciones en las que nos desplazamos en coche y por las que no andamos ni interactuamos con los demás o en bloques de pisos en los que no conocemos casi a ningún vecino.

Por otro lado vivimos un proceso de empobrecimiento social ${ }^{51}$. Las familias extensas se han atomizado y separado siendo cada vez más frecuentes los hogares de personas que viven solas. Podemos afirmar que las redes sociales reales de familia y amigos se han debilitado ${ }^{52}$ de forma global.

Es común que tengamos cada vez más contactos virtuales en redes sociales diversas y cada vez menos personas de carne y hueso en nuestros ámbitos íntimos reales. Esto modifica nuestro espectro relacional y en consecuencia la conducta

\footnotetext{
${ }^{50}$ Franganillo, J.. La ansiedad informativa. Uno, 2010; 5 abril:14.

${ }^{51}$ Pessacq, M. I., Transformaciones territoriales y empobrecimiento. Margen: revista de trabajo social y ciencias sociales, 2010; (58): 11.

${ }^{52}$ Almagiá, E. B. Apoyo social, estrés y salud. Psicología y salud, 2014; 14 (2): 237-243.
} 
social $^{53}$. Es lo que se denomina espejismo de las redes sociales ${ }^{54}$ donde nos creemos conectados a muchos gozando de cierto prestigio y posición virtual que en muchos casos no es más que ruido y humo.

La sociedad sufre una epidemia de soledad y aislamiento sin precedentes. Los niños pasan mucho tiempo sin sus padres o abuelos consumiendo contenido audiovisual o videojuegos. Los ancianos ${ }^{55}$ pasan mucho tiempo solos sin compañía de ningún tipo. Las madres y padres pasan mucho tiempo criando en soledad a sus hijos y trabajando en soledad cerrando un círculo que termina afectando a una gran mayoría ${ }^{56}$.

Es requisito fundamental para poder conectar con los demás haber conectado primero con uno mismo ${ }^{57}$. La conexión interior requiere de silencio como ya hemos visto. De ese silencio personal nacerá el suficiente espacio que permita una escucha verdadera, pilar y cimiento de toda comunicación de calidad.

Silenciar los procesos personales y despejarlos de ruido y prisa ${ }^{58}$ sería una buena recomendación a seguir.

\section{CONCLUSION}

La sociedad del conocimiento en que vivimos se caracteriza por el alto nivel de ruido de fondo que produce secundario al impacto que el urbanismo, la tecnología y los medios de comunicación producen en nosotros.

Esta sobrecarga de ruido e información es causa de malestar. Hemos analizado cómo el mundo sanitario y el docente son dos ejemplos de profesiones

\footnotetext{
${ }^{53}$ Campos Freire, F. Las redes sociales trastocan los modelos de los medios de comunicación tradicionales. Revista Latina de comunicación social, 2008; 11 (63).

${ }^{54}$ https://elpais.com/cultura/2015/12/30/babelia/1451504427_675885.html

${ }^{55}$ Rodríguez Martín, M. La soledad en el anciano. Gerokomos, 2009; 20 (4): 159-166.

${ }^{56}$ Del Olmo, C. ¿ Dónde está mi tribu?: maternidad y crianza en una sociedad individualista. Editorial Clave Intelectual, 2016.

${ }^{57}$ Vallejo-Nágera, J. A. Conócete a ti mismo. Temas de hoy, 1997.

${ }^{58}$ Honoré, C. Elogio de la lentitud. RBA Coleccionables, 2007.
} 
donde el ruido puede ser muy negativo tanto para los trabajadores como para sus usuarios.

En general las crisis vitales se configuran como el principal eje pedagógico personal a la hora de aprender a manejar el nivel de ruido personal y la desazón interna. Es el sufrimiento propio quien nos obligará a adaptarnos y para ello será preciso tener habilidades para construir silencio de calidad.

La epidemia de soledad social ${ }^{59}$ de la civilización globalizada es uno de los efectos del progreso que a día de hoy solo se combate generando ruido y distracción. Parece que va siendo necesario poner sobre el tapete la necesidad de que es preciso un mayor nivel de consciencia y silencio interior para que se puedan crear encuentros humanos y relaciones de calidad

Concluimos que la sobrecarga informativa ${ }^{60}$ y el ruido de fondo que produce son importantes fuentes de sufrimiento independientemente de la edad y demás condicionantes sociales. Dado que la sociedad actual produce mucho ruido de fondo multicanal es básico mejorar las habilidades individuales para generar silencio y aprender a relacionarnos con él. Cuidar los espacios de silencio personal e implementar actitudes meditativas que mejoren el contacto interior y las relaciones humanas de calidad son cursos de acción inteligentes que muchas tradiciones culturales defienden desde antaño y que cada cual podrá rescatar según sus valores y criterio.

La calibración ${ }^{61}$ y afinamiento personal y profesional precisan pues de espacio vacío interior por lo que aprender a equilibrar el ruido y el silencio en nuestra vida es un reto vital para todos.

\footnotetext{
${ }^{59}$ De Ussel, J.I. (2001) La soledad en las personas mayores: Influencias personales, familiares y sociales: Análisis cualitativo. Ministerio de Trabajo y Asuntos Sociales, Secretaría General de Asuntos Sociales, Instituto de Migraciones y Servicios Sociales.

${ }^{60}$ Serrano Puche, J. (2017) Por una dieta digital: hábitos mediáticos saludables contra la "obesidad informativa". Ámbitos, no 24.

${ }^{61}$ Oliver, E. B.; De Albornoz, P. Arranz Carrillo; López. H.C. Herramientas para el autocuidado del profesional que atiende a personas que sufren. FMC-Formación Médica Continuada en Atención Primaria, 2011; 18 (2): 59-65.
} 
Silencio en el ruido: oportunidades para la meditación en la vida cotidiana Salvador Casado

Como bien saben los poetas, la inspiración depende de las musas. Dado que estas gustan de habitar lugares alejados, atrevámonos a navegar silencios para llegar a ellas. Es un viaje arriesgado ${ }^{62}$ pero podemos adelantar que merece la pena.

\section{Salvador Casado}

Médico de Medicina Familiar y Comunitaria.

Centro de salud de Villalba en la Comunidad de Madrid.

E-mail: vigidel@gmail.com

https://www.doctorcasado.es/

\section{Cómo citar este artículo:}

Casado, S., "Silencio en el ruido: oportunidades para la meditación en la vida cotidiana", en Folia Humanística, 2018; 9: 21-40. Doi: http://dox.doi.org/10.30860/0039.

(C) 2018 Todos los derechos reservados a la Revista Folia Humanística de la Fundación Letamendi Forns. This is an open access article

${ }^{62}$ Homero; Pabón, J. M.. (2005) Odisea. Ed. El Tiempo. 